\title{
A Cerebellopontine Angle Epidermoid Cyst Presenting as Trigeminal Neuralgia: A Case Report
}

\author{
Nilofer Halim*, Chaithra Kalkur and Anusha L Rangare \\ Reader, Department of Oral Medicine and Radiology, Century International Institute of Dental Sciences and Research Centre, Kerala, India \\ *Corresponding author: Nilofer Halim, Reader, Department of Oral Medicine and Radiology, Century International Institute of \\ Dental Sciences and Research Centre, Kerala, India
}

\begin{abstract}
Trigeminal neuralgia is the one of the most painful condition known to mankind, so much so that it has described as suicide illness. The diagnosis of Trigeminal neuralgia is clinically plausible due to its specific characteristics. Differentiating Classical form secondary Trigeminal neuralgia requires further evaluation with either a CT or MRI. A correct diagnosis is crucial, as patients can then follow a generally acknowledged treatment modality of either Medication or surgery. The aim of this article is to present a case of Trigeminal Neuralgia secondary to Cerebellopontine angle Epidermoid cyst in an elderly female patient. She had to be maintained with medicines alone as she was skeptical of undergoing brain surgery. This report also portrays that secondary trigeminal neuralgia can be managed long term with carbamazepine without major adverse effects.
\end{abstract}

Keywords: Epidermoid Cyst; Cerebellopontine Angle; Trigeminal Neuralgia

\section{Introduction}

Trigeminal neuralgia is the one of the most painful condition known to mankind, so much so that it has described as suicide illness [1]. The diagnosis of trigeminal neuralgia is clinically plausible due to its specific characteristics. According to leading researcher of trigeminal neuralgia, Prof. Joanna Zakrzewska's, it is one of the few chronic pain conditions in which sufferers can be rendered pain-free either with medications or surgery [2]. Therefore, correct diagnosis is crucial, as patients can then follow a generally acknowledged treatment modality. The aim of this article is to present a case of Trigeminal Neuralgia secondary to Cerebellopontine angle Epidermoid cyst in an elderly female patient, who has refused undergoing surgery and had to be maintained with medicines alone. This also portrays that secondary trigeminal neuralgia can be managed long term with carbamezapine without major adverse effects.

\section{Case Report}

A 50-year-old female patient presented to the department of oral medicine and radiology, at Century dental college with complaint of excruciating pain in the lower right side of the face for one year. The pain was sudden in onset, sharp shooting in nature and lasted only for a few seconds. The episodes were frequent and occurred many times in a day. She had sought treatment at various dental clinic during which time most of her teeth on the right side were extracted (Figure 1). She had applied a skin oil for pain intraorally two days back which had resulted in chemical burn. Her medical history was significant, that she was under treatment for epilepsy and suffered with herpes zoster and was treated with acyclovir $800 \mathrm{mg}$ for 7 days. She was conscious and cooperative, of short stature and moderately nourished. There was pallor of the skin and inferior palpebra. Intraorally, pallor was noticeable on the buccal mucosa, erosion was detected on the lower right alveolar ridge region in relation to the missing premolars. The upper and lower arches were partially edentulous. Trigger zone was elicited at the right lower border of the mandible. A provisional diagnosis of Trigeminal neuralgia was made. CT scan of the brain showed a heterogenous lesion with cystic areas and calcification in right Cerbello Pontine angle extending to the medial aspect of temporal lobe. There is compression and rotation of brain stem to the left. An impression of CP angle neoplasm was given on CT (Figure 2). 

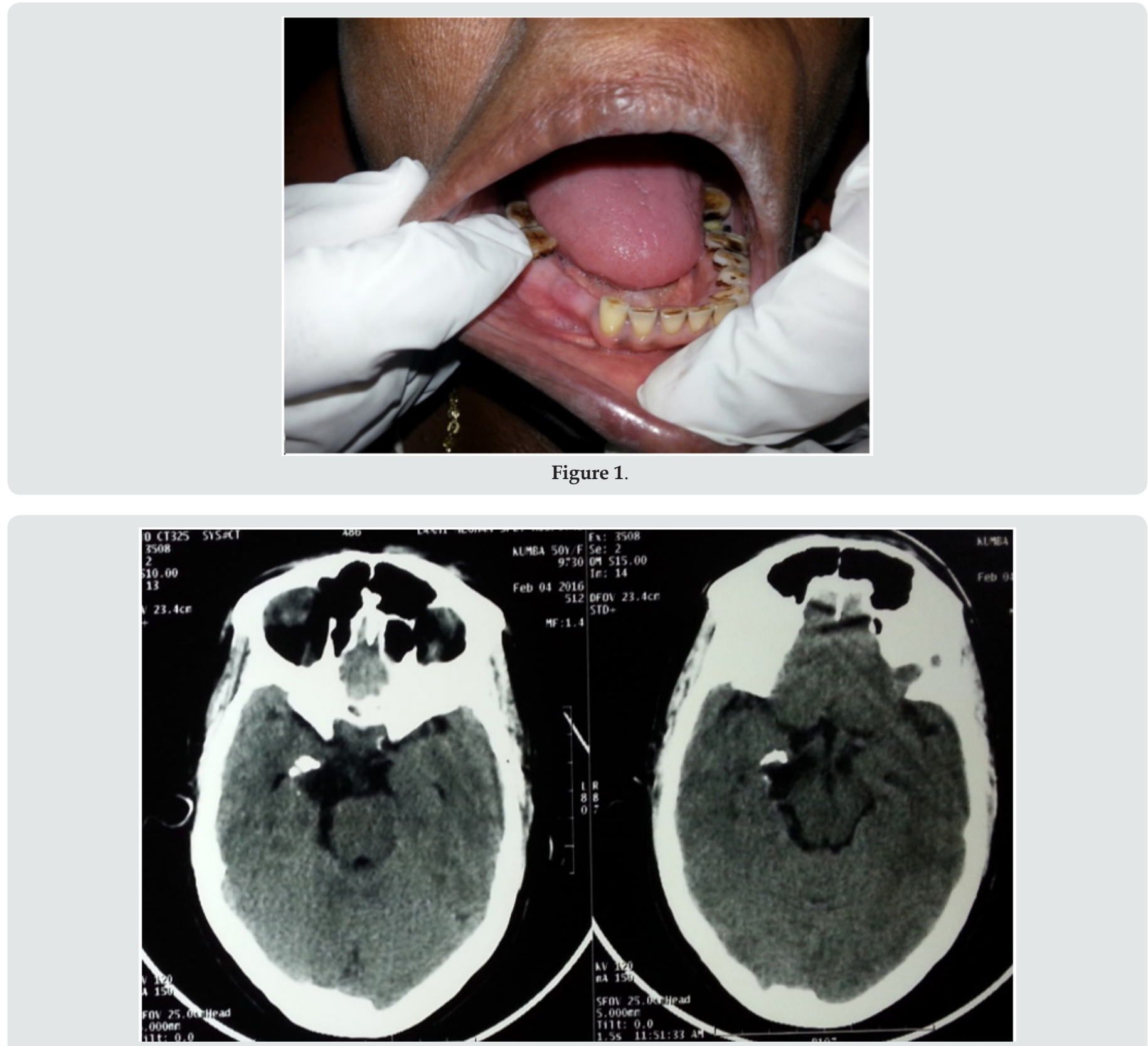

Figure 2.

MRI of the brain revealed a lesion of size $15 \times 33 \times 30 \mathrm{~mm}$ involving the right CP angle cistern, which appeared hypointense in T1 (Figure 3a) and hyperintense in T2 image. It was causing compression of pons and lower midbrain. The $3^{\text {rd }}$ and $6^{\text {th }}$ cranial nerves were compressed and encased by the lesion with displacement towards left. $7^{\text {th }}$ and $8^{\text {th }}$ cranial nerves were closely abutted (Figure 3b). MR study was suggestive of epidermoid cyst involving right basal cistern and $\mathrm{CP}$ angle cistern. Based on these findings, diagnosis of Trigeminal neuralgia secondary to epidermoid cyst located at right cerebella pontine cistern was made. Patient was prescribed carbamezapine $200 \mathrm{mg}$ twice daily gradually increased to $400 \mathrm{mg}$ tid. She was referred to neurologist who advised surgery for the brain lesion. Patient and her family members were very apprehensive about surgery and did not want to proceed with surgery. She has been on medication for the past 2 years and the dose of carbamezapine was reduced to $100 \mathrm{mg}$ twice daily. Regular blood test has been done to check for myelosuppression. There was a single episode of unbearable pain, for which inferior alveolar nerve block was given to arrest the pain. Long term treatment follows up of the patient can be described as favorable as she has been able to carry on normal routine with only few occasional episodes of severe pain. 


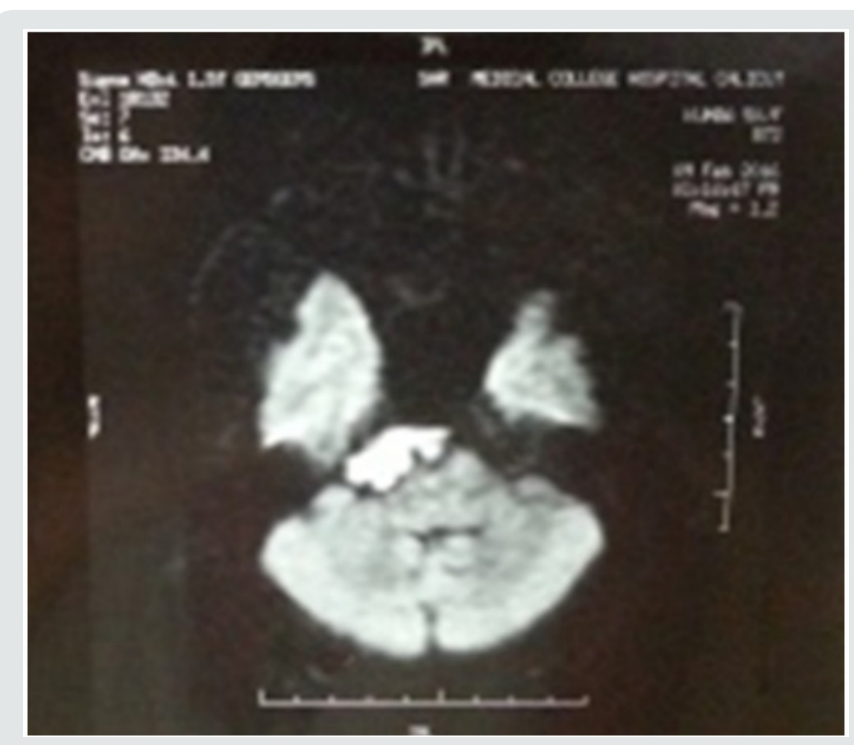

Figure 3a.

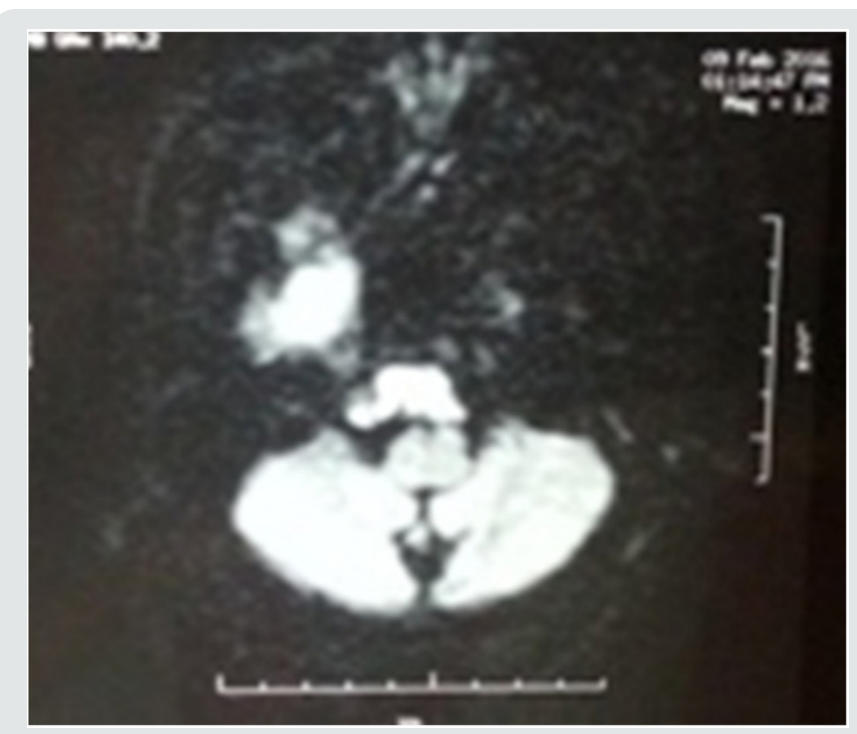

Figure $3 b$.

\section{Discussion}

Trigeminal neuralgia is a neuropathic pain with distinct diagnostic criteria. The IASP definition of trigeminal neuralgia (TN) is "sudden, usually unilateral, severe, brief, stabbing, recurrent episodes of pain in the distribution of one or more branches of the trigeminal nerve." Trigeminal neuralgia is a rare condition [2]. The peak incidence is in the age group of $50-60$ with preponderance in women [3]. However, some Japanese and Chinese reports have quoted a male predominance in those above 80 years of age [4]. It is a condition that has been recognized many centuries ago, The French terminology Tic douloureux was coined by surgeon Nicolaus Andre [5]. There are two recognized forms of $\mathrm{TgN}$, Classical and Secondary or Symptomatic which is related either to central nervous system lesion or multiple sclerosis [4]. There is also Atypical form which does not have clearly definable features and symptoms may overlap that of atypical facial pain. This has been described as atypical trigeminal neuralgia4. The aetiology of the condition is still unclear5. Most researchers suggest that root entry zone (REZ) of the trigeminal ganglion to be site of generation of pain. Compression of the REZ by blood vessels or tumors and demyelination are the most common abnormality found in this area. This leads to hyperexcitability and central sensitization $[2,4,5]$.

Trigeminal neuralgia is a clinical diagnosis. The characteristic features being

a) Paroxysmal (abrupt onset and termination) attacks of pain lasting for a few seconds to few minutes

b) It involves one or more division of trigeminal nerve, unilaterally.

c) Character is described as sharp, shooting, stabbing or burning.

d) Severe intensity.

e) Presence of Trigger zones (stimulus evoked: palpation by routine activity such as touching, washing the face, brushing teeth) intensifies pain usually found around the nose or mouth.

f) Presence of refractory period.

The two accepted modalities for treatment are medical and surgical.

\section{Medical Management}

Gold standard for medical treatment is response of the condition to Carbamazepine. Carbamazepine is a tricyclic imipramine first synthesized in 1961 and introduced for treatment of trigeminal neuralgia by Blom [5,6]. It is usually started at $200 \mathrm{mg}$ per day single dosing and gradually increased to up to $800 \mathrm{mg}$ i.e. four divided doses of $200 \mathrm{mg}$ each. The most common side effect is hypersensitivity ( $5-10 \%$ of the patient), folic acid deficiency and megaloblastic anemia6. Drug interaction with warfarin has also been reported. Oxycarbazepine, is a daughter drug and has fewer side effects. A $300 \mathrm{mg}$ is equipotent to $200 \mathrm{mg}$ of carbamazepine. The improved safety profile makes it a better option to use. Lamotrigine, Gabapentin and Baclofen are other second-line drugs that could be prescribed $[2,5,7]$.

\section{Surgical Procedures}

Various surgical procedures have been conducted and they can be briefly classified into

a) Peripheral surgery such as Neurectomies, Cryotherapy, Laser, Radiofrequency Thermo-Rhizotomy and Injections with Streptomycin, Alcohol, Glycerol and Phenol. Analgesic blocking of peripheral receptors also arrests pain. This effect is also diagnostic [8].

b) Surgery at Gasserion Ganglion: Percutaneous radiofrequency rhizotomy /thermocoagulation and Percutaneous glycerol rhizolysis. These procedures are less invasive and are associated with low rates of mortality and morbidity. However, they are associated with anesthesia dolorosa, facial numbness and dysesthesia [8]. 
c) Surgery at the REZ: Microvascular decompression is one of the most successful procedures, providing relief for $70 \%$ of patients for up to 10 years. It is a nondestructive procedure. This procedure involves major neurosurgery and is therefore not suitable for all patients; it carries a mortality rate of $0.5 \%$ and a $2 \%$ risk of hearing loss [9]. Gamma knife surgery is a noninvasive radiosurgery [8].

All forms of surgery have potential for complications with sensory loss being the most common one. Recurrence of pain within 1- 4 years occurs with most of the procedures. The reported range for TN due to tumors is $0.8 \%-11.6 \%$ [10]. Tumors leading to TN are mostly benign and typically compress the root near its entry into the pons. Epidermoid tumors are slow growing, and symptoms also appear later in the course, it is also referred to as Cholesteatoma [11]. Patients usually present in 4- 5th decade of life Hearing loss is the most common presenting sign followed by trigeminal neuralgia. Other neurological deficits include facial paresis, hearing disturbances and third and sixth nerve palsy. It has been suggested that if pain occurs bilaterally or there is simultaneous involvement with other nerve trunk then a systemic involvement or expanding tumor has to be suspected [12]. Pathogenesis of $\mathrm{TgN}$ in epidermoid is uncertain, however it has been attributed to either direct compression or displacement of the nerve at REZ. It has been reported that neuralgia due to epidermoid tumors are clinically indistinguishable to classical $\mathrm{TgN}$. However, the age of onset in this condition is earlier than the Classical TgN [12]. In this case the symptoms started at the age of 49 years and were confined to mandibular branch of trigeminal nerve only, it remained rather consistent with only sporadic flareup. This may be attributed to the medical treatment (carbamzepine).

On CT scans they are homogenous density of an epidermoid cyst enables it to be distinguished from other tumors [12]. In MRI they have lamellated or onion skin appearance. They have low or intermediate intensity in $\mathrm{T} 1$ weighted images and high intensity in $\mathrm{T} 2$ weighted images13. MRI is the preferred imaging modality to visualize the anatomic landmarks around the trigeminal ganglion and the $\mathrm{CP}$ angle as it gives the best soft-tissue resolution and excellent visualization of the intracranial and extracranial course of the nerve $[12,13]$. They have to be differentiated from Schwannomas, meningiomas or chondromas by signal intensity criteria [13]. Surgically the cyst appears as white and pearly with encasement or compression of the trigeminal nerve. Optimum treatment is radical removal of the complete tumor [14]. This is problematic in most cases as it has wide extension and firm adhesion to neurovascular structures. To minimize neurologic deficits a part of the tumor is left behind. Other complications that are reported are meningitis, cerebellar and brain stem infarction. The possible REZ arterial compression is treated with an additional Microvascular decompression. $0-30 \%$ estimated recurrence rate have been reported in long follow up studies [14]. One of the main aspects regarding trigeminal neuralgia as highlighted by Drangsholt and Truelove is the diagnosis of this condition [15]. Majority of patients attending the clinic have previously been misdiagnosed and had undergone irreversible dental treatment, even when they had presented with classical features of TN, as in this case.

\section{Conclusion}

William Osler once said, "Listen to the patient: he is telling you the diagnosis." There are no objective diagnostic tests for idiopathic TNA. As a part of the diagnostic work-up, patients should have a magnetic resonance imaging (MRI) scan or a computed tomography scan to rule out secondary $\mathrm{TN}$. If detected, these lesions are best treated surgically. although surgical procedures do carry risk of significant morbidity. However, convincing the patients regarding surgical treatment may be difficult as in this case report. Patients should be treated with carbamezapine as a first line therapy, followed by addition of other agents if patients become unresponsive. The patients must be followed up for long term for any adverse effects.

\section{Funding}

This research did not receive any specific grant from funding agencies in the public, commercial or not for profit sectors.

\section{References}

1. JM Zakrzewska, Harrison S (2002) Assessment and management of Orofacial pain: Pain research and clinical management. vol 14.

2. JM Zakrzewska, McMillan R (2011) Trigeminal neuralgia: the diagnosis and management of this excruciating and poorly understood facial pain. Postgrad Med J 87: 410-416.

3. Bennetto L, NK Patel, Fuller G (2007) Trigeminal neuralgia and its management. BMJ 334: 201-205.

4. Cruccu G, Finnerup BN, Jensen TS, Scholz J, Nurmikko T, et al. (2016) Trigeminal neuralgia New classification and diagnostic grading for practice and researchNeurology 87(2): 220-228.

5. BM Zussman, Moshel Yaron A (2012) Trigeminal neuralgia: case report and Review. JHN Journal 7(2): 2-5.

6. Taylor JC, Brauer S (1981) Long term treatment of trigeminal neuralgia with carbamazepine. Postgraduate medical Journal 57: 16-18.

7. Saraswathi GK, Swathi T (2017) Trigeminal Neuralgia - A Case Report with Review of Literature. SAJ Case Report 4: 102

8. Al Quluti (2015) Update on neuropathic pain treatment for trigeminal neuralgia. The pharmacological and surgical options. Neurosciences 20(2): 107-114

9. Oesman C, Mooij JJ (2011) Long term follow up of microvascular decompression for trigeminal neuralgia. Skull base 21(5): 313-322.

10. K Toda (2007) Etiology of Trigeminal neuralgia. oral science international 4(1): 10-18.

11. Smiriniotopoulos GJ, Yue NC, Rushing EJ (1993) Cerebellopontine angle masses: Radiologic - Pathologic Correlation. Radio Graphics 13: 1131147.

12. KS Guttal, VG Naikmasur, SK Joshi, RJ Bathi (2009) Trigeminal neuralgia secondary to epidermoid cyst at the cerebellopontine angle: case report and brief overview. Odontology 97(1): 54-56.

13. Goh BT, Poon CY, Peck RH (2001) The importance of routine magnetic resonance imaging in trigeminal neuralgia diagnosis. Oral Surg Oral Med Oral Pathol Oral Radiol Endod 92(4): 424-429.

14. DW Son, Chang Hwa Choi, Seung Heon Cha (2010) Epidermoid tumors in the Cerebellopontine angle presenting with trigeminal neuralgia. J Korean Neurosurg Soc 47(4): 271-277.

15. JM Zakrzewska, Padfield D (2014) The patients' journey through trigeminal Neuralgia. Pain clinical Updates 22(1). 
(c) (P) This work is licensed under Creative

To Submit Your Article Click Here: Submit Article

DOI: $10.32474 /$ SJO.2019.02.000144

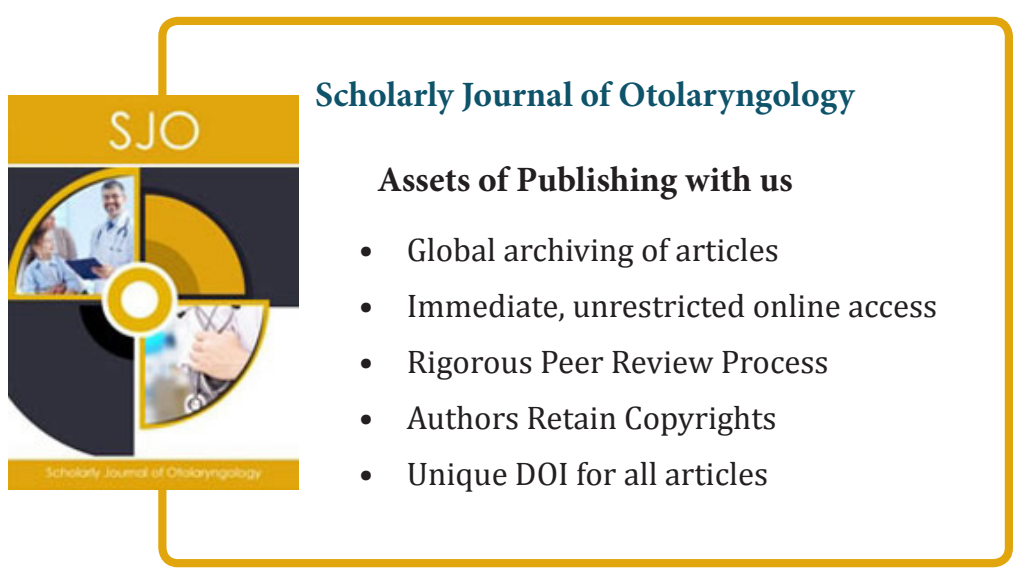

